\title{
Star Formation Law at Sub-kpc Scale in the Elliptical Galaxy Centaurus A as Seen by ALMA
}

\author{
Jazeel H. Azeez, ${ }^{1,2}$ Zamri Z. Abidin, ${ }^{1}$ C.-Y. Hwang, ${ }^{3}$ and Zainol A. Ibrahim ${ }^{1}$ \\ ${ }^{1}$ Physics Department, Faculty of Science, University of Malaya, 50603 Kuala Lumpur, Malaysia \\ ${ }^{2}$ Physics Department, Faculty of Science, AL-Nahrain University, Baghdad 10072, Iraq \\ ${ }^{3}$ Graduate Institute of Astronomy, National Central University, Chung-Li 32054, Taiwan \\ Correspondence should be addressed to Jazeel H. Azeez; jazeelhussein@yahoo.com
}

Received 28 January 2017; Accepted 19 April 2017; Published 13 June 2017

Academic Editor: Dean Hines

Copyright (C) 2017 Jazeel H. Azeez et al. This is an open access article distributed under the Creative Commons Attribution License, which permits unrestricted use, distribution, and reproduction in any medium, provided the original work is properly cited.

\begin{abstract}
We present an extensive analysis of the relationship between star formation rate surface density $\left(\sum\right.$ SFR $)$ and molecular gas surface density $\left(\sum \mathrm{H}_{2}\right)$ at sub-kpc scale in the elliptical galaxy Centaurus A (also known as NGC 5128) at the distance $3.8 \mathrm{Mpc} .{ }^{12} \mathrm{CO}(J=$ 2-1) data from Atacama Large Millimetre/Sub-Millimetre Array SV data with very high resolution $\left(2.9^{\prime \prime}, 0.84^{\prime \prime}\right)$, as well as $24 \mu \mathrm{m}$ data from the Spitzer Space Telescope, were used. This is one of the first studies of the SF law on Centaurus A at this very high spatial resolution. The results showed a breakdown in star formation law with a $0.49 \pm 0.05$ index relating $\sum$ SFR and $\sum \mathrm{H}_{2}$ at $185 \mathrm{pc}$. A significant correlation exists between surface densities of molecular gas and SFR with very long depletion time (68 Gy). In addition we examined the spatially resolved relationship between velocity dispersion and star formation rate surface density for the outer disk of this galaxy and we found that the average velocity dispersion is equal to $11.78 \mathrm{~km} / \mathrm{s}$. The velocity dispersion of the molecular ISM for the outer disk is found to follow a power relation with the star formation rate surface density $\sigma \propto\left(\sum \text { SFR }\right)^{\beta}$, where $\beta$ is the slope from the ordinary least square fitting. The value of $\beta$ is about $1 / n \approx 2.16 \pm 0.40$ and $n$ is the power law index of the star formation law.
\end{abstract}

\section{Introduction}

The present challenge with elliptical galaxies is to resolve their formation and evolutionary history. Elliptical galaxies are currently known to contain an interstellar medium (ISM) that varies from that in a spiral galaxy [1]. Observations have previously detected cold gas, dust, and even new/residual star formation in elliptical galaxies [2-5]. It is well known that stars are formed from molecular gas, so studying the molecular gas in galaxies provides important indications of the primary physical process of massive star formation in galaxies [6]. The molecular gas in early-type galaxies was studied by many authors such as [7-10].

To understand star formation in galaxies, the relations between star formation rate surface density $\sum$ SFR and gas surface density $\sum$ gas must be determined. Schmidt [11] put forth a power law that presents a correlation between $\sum$ SFR and $\sum$ gas: $\sum$ SFR $\propto \sum$ gas $^{N}$. Kennicutt Jr. [12, 13] proved that the Schmidt law relates gas density to star formation density within many orders of magnitude and determined a power law relationship with index $N=1.4$. This relationship is usually called the Kennicutt-Schmidt (K-S) law. The most recent studies on the K-S law examined the index at subkpc scales [14-17], closing from the intrinsic scale of star formation, that is, giant molecular clouds size (GMCs) [6]. Kennicutt \& Evans [18] reviewed the relation between the star formation at different scale from entire galaxies to individual molecular clouds; at sub-kiloparsec scale they recognized two regimes, the first with low density subthreshold regime and the second with high density regime. They found that the SFR density correlates well with the gas density for the second regime, while it is uncorrelated for the first one. The theories that describe and interpret the star formation process in these two regimes were studied by McKee \& Ostriker [19].

One of the most interesting nearby galaxies is NGC 5128 (Centaurus A) (see [20], for a review of the properties of Cen A), the closest, most easily observable giant elliptical galaxy [21]. It is the dominant object in the Centaurus A group [22] 
and lies at a distance of 3.8 Mpc [23-25]. This distance (where $1^{\prime \prime}$ is approximately $18.5 \mathrm{pc}$ ) provides a unique opportunity to study the molecular gas in extraordinary detail. NGC 5128 shows considerably clear dust lanes that are probably caused by merger activities in the past (see [26, 27]). The molecular interstellar medium in the dust lane has been widely studied [28-36]. In addition, due to it being a strong source of radio and far infrared and X-ray emissions, it has been investigated in detail over a wide part of observable spectrum [37]. CO mapping reveals that the disk contains $2 \times 10^{8} M_{\odot}$ of molecular gas [29].

Rare studies covering the K-S law in Centaurus at subkpc scale have been reported. How do the $\mathrm{SFR}$ and $\mathrm{H}_{2}$ surface densities relate to each other on sub-kpc spatial resolution in an elliptical galaxy? To address this question, we determine $\sum \mathrm{H}_{2}$ in the central area of the elliptical galaxy Centaurus A by adopting high spatial resolution (185 pc).

\section{Data}

Observations of the Centaurus A in ${ }^{12} \mathrm{CO}(J=2-1)$ emission line were undertaken from August 11, 2011, at 19:19 (UTC) to August 12, 2011, at 01:00 (UTC) by using a mosaic of 48 pointing with a total integration time of $\sim 341.5 \mathrm{~min}$. These public data are available at the Atacama Large Millimetre/Submillimeter Array (ALMA) Science Portal (https://almascience.nrao.edu/alma-data/science-verification). Observations were conducted in band $6(\sim 1.3 \mathrm{~mm})$. Four spectral windows were used, each with a $1.88 \mathrm{GHz}$ bandwidth, 256 channels, and unique polarization. The data have a rms of $2.66 \mathrm{mJy}$ with a spectral resolution of $20 \mathrm{~km} \mathrm{~s}^{-1}$. The observation parameters are listed in Table 1 . The data were reduced, and images were processed using the Common Astronomy Software Application package (CASA). The continuum was first subtracted in the visibility domain. The images were processed using the CLEAN algorithm with a robust $=0.5$ weighting (Briggs weighting) of the visibilities. The $\mathrm{CO}$ data were binned spectrally into channels with $20 \mathrm{~km} \mathrm{~s}^{-1}$ wide. The procedure is described in the scripts available on the ALMA Science Portal (https://almascience.nrao.edu/almadata/sciver/CenABand6/).

To estimate the amount of star formation obscured by dust, we used $24 \mu \mathrm{m}$ data obtained by SPITZER (MIPS). The data was obtained from the public archive (http://sha.ipac .caltech.edu/applications/Spitzer/SHA/). The resolution element (beam size) of MIPS band is $\sim 6^{\prime \prime}$, with pixel size of $2.55^{\prime \prime}$. The $24 \mu \mathrm{m}$ data has a $\sim 5^{\prime}$ square field of view.

\section{Results and Discussion}

3.1. CO Maps and Line Emission Distribution. The maps of the integrated intensity and velocity dispersion of the emission line ${ }^{12} \mathrm{CO}(J=2-1)$ are shown in Figures 1 and 2 . We can see from Figure 1 that the distribution can be divided into three regions: a central emission region and two regions (northeast, $\mathrm{NE}$, and southwest, SW, one on either side of the centre) forming an S-like shape. The NE region takes an arc shape
TABLE 1: ALMA observational parameters.

\begin{tabular}{lc}
\hline Parameter & Value \\
\hline Target & Centaurus A (CenA, NGC 5128) \\
Observing date & 2011 August 11, 12 \\
Total integration time & $\sim 341.5 \mathrm{~min}$ \\
Field center: & $12^{\mathrm{h}} 25^{\mathrm{m}} 27.61^{\mathrm{s}}$ \\
R.A. $\left(\mathrm{J}_{2000}\right)$ & $-43^{\circ} 01^{\prime} 08.8^{\prime \prime}$ \\
Dec. $\left(\mathrm{J}_{2000}\right)$ & 15 \\
Number of antenna & $542 \pm 7 \mathrm{~km} \mathrm{~s}^{-1}$ \\
System velocity & $230 \mathrm{GHz}^{\prime}$ \\
Rest frequency & $\left(\mathrm{Major}, \mathrm{minor}^{\mathrm{P} . \mathrm{A} .}\right)$ \\
Restoring beam & $\left(2.9^{\prime \prime}, 0.84^{\prime \prime}, 82.65^{\circ}\right)$ \\
& $1.88 \mathrm{GHz}^{\circ}$ \\
Total bandwidth & 256 \\
Total channels & $20 \mathrm{~km} \mathrm{~s}^{-1}$ \\
Velocity resolution & $6328.09 \mathrm{Jy} \mathrm{km} \mathrm{s}^{-1}$ \\
Integrated flux density & 0.00183 \\
Redshift $(z)$ &
\end{tabular}

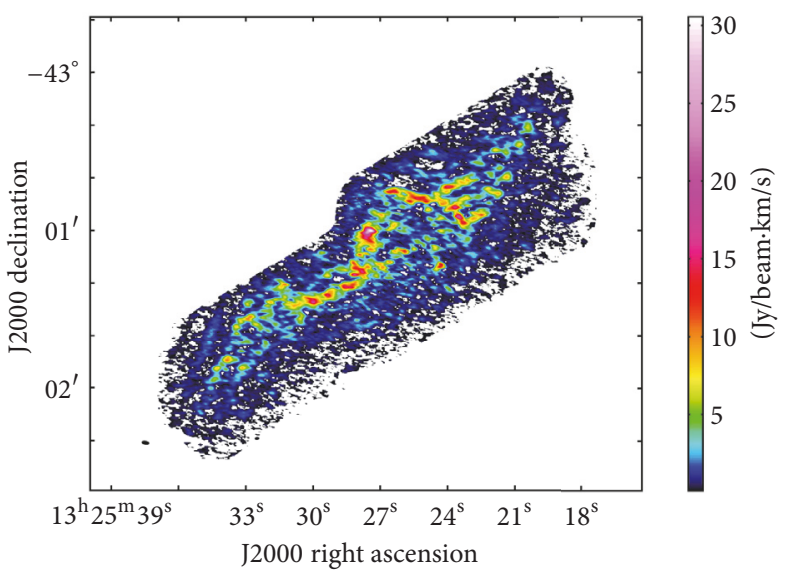

Figure 1: ALMA ${ }^{12} \mathrm{CO}(J=2-1)$ integrated intensity images of Centaurus A (NGC 5128).

that extends to the central region while the SW region takes a semiarc shape that extends from the central region and becomes fainter as it goes radially outwards. It is clear that the NE region is brighter than the SW region because the latter is $\mathrm{CO}$ poorer. These two regions have also been identified by Bland et al. [38], who observed ionized gas with Centaurus A. His results revealed that the HII regions were embedded in a faint component of diffusely ionized gas and confined to an envelope which has the form of a hysteresis loop. From the same figure it is noticed that the CO distribution is centrally peaked, and this is also observed in most Sc spiral galaxies $[39,40]$.

3.2. Molecular Gas Mass and Surface Density. The mass of the molecular gas was calculated from the ${ }^{12} \mathrm{CO}(2-1)$ emission 


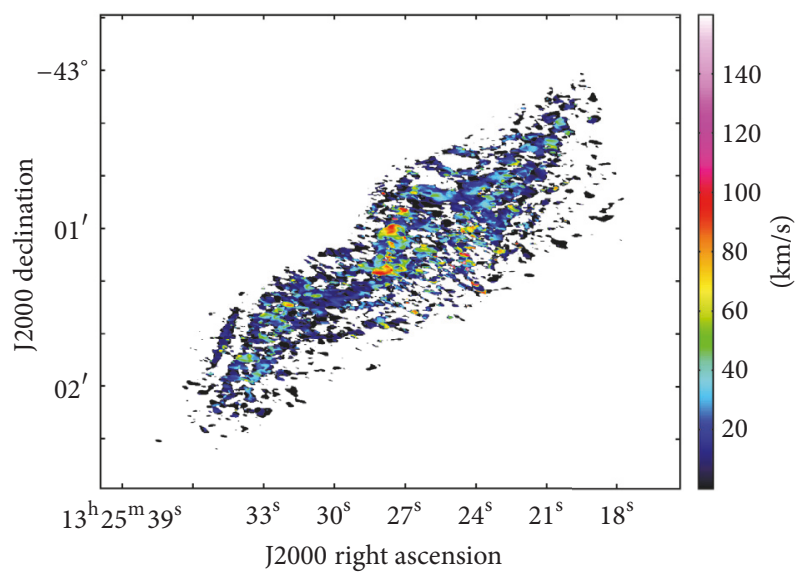

Figure 2: ALMA ${ }^{12} \mathrm{CO}(J=2-1)$ velocity dispersion images of Centaurus A (NGC 5128).

line as tracer for molecular hydrogen gas using the following formula:

$$
\left(\frac{M_{\mathrm{H}_{2}}}{M_{\odot}}\right)=3 \cdot 92 \times 10^{-17} X_{\mathrm{CO}}\left(\frac{S_{\mathrm{CO}}}{\mathrm{Jykm} \mathrm{s}^{-1}}\right)\left(\frac{D}{\mathrm{Mpc}}\right)^{2} .
$$

We adopt a $\mathrm{CO}(2-1) / \mathrm{CO}(1-0)$ line ratio $\left(R_{21}\right)$ of 0.55 [41]. The molecular hydrogen mass has been calculated in a region of $(\sim 1.5 \mathrm{kpc})$ diameter to be $\sim 2 \times 10^{8} M_{\odot}$ by adopting the distance $D=3.8 \mathrm{Mpc}$. By comparing the data with the single dish [28] value of $1 \times 10^{9} M_{\odot}$ (with $D=7 \mathrm{Mpc}$ ), a difference of $33 \%$ is found by using the same distance. This difference is attributed to missing flux, because this data does not contain short spacing information (ACA and single dish), in addition the relatively short observation time and the high uncertainties in data calibration for the mentioned single dish may also participate in increasing the missing flux. In the present study, the central region of Centaurus A was divided into many boxes each one with angular resolutions of $10^{\prime \prime} \times 10^{\prime \prime}$. The angular to linear scale is $1^{\prime \prime}=18.5 \mathrm{pc}$; thus, these boxes correspond to the $185 \mathrm{pc} \times 185$ scale. In each box the molecular gas mass surface density was calculated using conversion factor $X_{\mathrm{CO}}=2 \times 10^{20} \mathrm{~cm}^{-2}\left(\mathrm{~K} \mathrm{~km} \mathrm{~s}^{-1}\right)^{-1}[42,43]$. The molecular gas mass and surface density of each region are listed in Table 2, which shows that some regions have huge amount of molecular gas mass $\left(1-14 \times 10^{6} M_{\odot}\right)$ this indicates that several GMC associations are located there [44].

3.3. Star Formation Rate Surface Density and Star Formation Law. The infrared data are used as star formation tracers. The infrared data are obtained from the Spitzer Space Telescope. To estimate the amount of star formation obscured by dust, we use $24 \mu \mathrm{m}$ data obtained from the public archive which is taken with the MIPS instrument. According to Helou [45] and Eckart et al. [29] the Centaurus A has cirrus like colours; however, its effect is weak on our data due to two reasons; the first is the used wavelength is $24 \mu \mathrm{m}$. Bendo et al. [46] made an overview of ancillary 24,70 , and $160 \mu \mathrm{m}$ data from the Multiband Imaging Photometer for Spitzer (MIPS) for

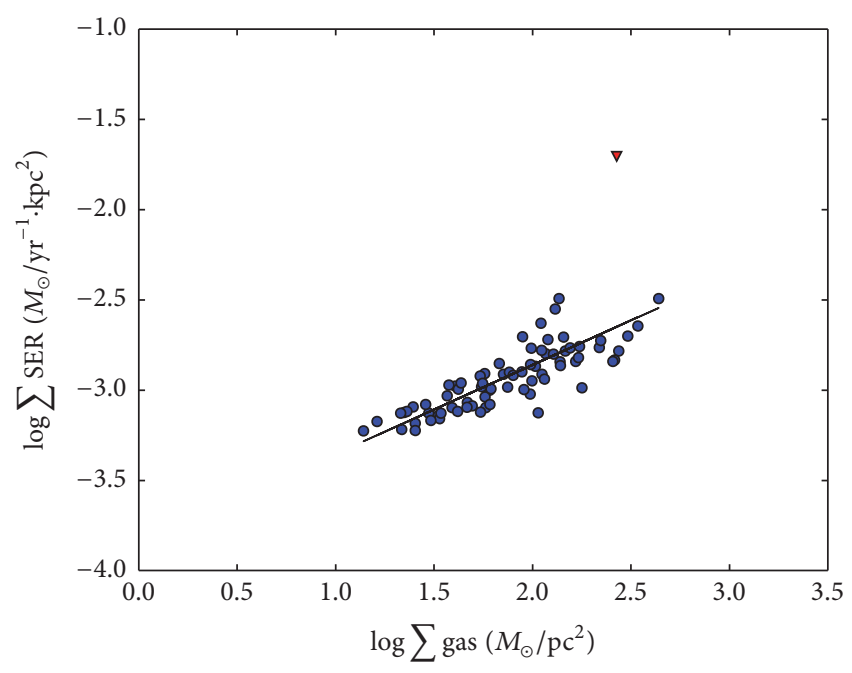

FIgURE 3: Relation between the surface density of molecular hydrogen $\sum \mathrm{H}_{2}$ and surface density of star formation rate $\sum \mathrm{SFR}$. The solid line represents the linear square fit line.

samples of nearby galaxies (including Centaurus A). In their study they concluded that the cirrus like colour in Centaurus $\mathrm{A}$ and in the other galaxies is only effective at the wavelengths 70 and $160 \mu \mathrm{m}$ and its effect is not influential in $24 \mu \mathrm{m}$. The second reason, as Eckart et al. [29] concluded, is that the cirrus clouds emission is due to cold dust with larger spatial extent than the molecular disk. In the current data we only focused on the molecular disk. Accordingly, the cirrus clouds emission outside the disk has no effect on the observed emission and as a result on star formation.

Wu et al. [47] showed that the SFR can be estimated from $24 \mu \mathrm{m}$ luminosity using the following formula:

$$
\frac{\sum \mathrm{SFR}}{M_{\odot} \mathrm{yr}^{-1}}=\frac{v L_{v}[24 \mu \mathrm{m}]}{6.66 \times 10^{8} L_{\odot}}
$$

where $L_{v}$ is the $24 \mu \mathrm{m}$ dust luminosity. The archival $24 \mu \mathrm{m}$ data from MIPS show that there is very weak star formation (few $10^{-5}-10^{-4} M_{\odot} \mathrm{yr}^{-1}$ ). The total SFR in this galaxy 3.89 $\times 10^{-3} M_{\odot} \mathrm{yr}^{-1}$ was found to be lower than the previous estimates. The relationship between the SFR and molecular gas surface densities which is known as star formation law or (Kennicutt-Schmidt law) is drawn for NGC 5128 with the spatial resolution of $10^{\prime \prime} \times 10^{\prime \prime}$ as shown in Figure 3. The derived values of star formation rate surface density at this spatial resolution are shown in Table $2 .{ }^{12} \mathrm{CO}(J=2-1)$ was used as a molecular gas density indicator. All data points from the boxes at this spatial resolution were combined to fit a line in log-log space using ordinary least square fitting (solid line in Figure 3). We found a high significant correlation with correlation coefficient 0.75 with a probability $p=100 \%$. The power law index of the $\sum$ SFR- $\sum \mathrm{H}_{2}$ relation for the elliptical galaxy NGC 5128 data is about $0.49 \pm 0.05$. This value is significantly lower than a typical power law index of the K$S$ law. In the Milky Way, young clusters are known to recede from their parents at a rate of $10 \mathrm{~km} \mathrm{~s}^{-1}$ [48]. As they evolve, they recede from their parent GMCs by a scale of $100 \mathrm{pc}$ over 
TABLE 2: CO intensity, molecular gas density and mass, star formation rate, and star formation rate surface density. With spatial resolution of $185 \mathrm{pc} \times 185 \mathrm{pc}$.

\begin{tabular}{|c|c|c|c|c|c|}
\hline Box & $\begin{array}{l}S_{\mathrm{CO}(2-1)} \Delta V \\
\left(\mathrm{Jy} \mathrm{km} \mathrm{s}^{-1}\right) \\
\end{array}$ & $\begin{array}{c}M\left(\mathrm{H}_{2}\right) \\
M_{\odot} \times 10^{6}\end{array}$ & $\begin{array}{c}\sum \mathrm{H}_{2} \\
\left(M_{\odot} \mathrm{pc}^{-2}\right)\end{array}$ & $\begin{array}{c}\text { SFR } \\
\left(M_{\odot} \mathrm{yr}^{-1}\right) \times 10^{-5} \\
\end{array}$ & $\begin{array}{c}\sum \mathrm{SFR} \\
\left(M_{\odot} \mathrm{yr}^{-1} \mathrm{kpc}^{-2}\right) \times 10^{-3}\end{array}$ \\
\hline 1 & 14.82 & 0.76 & 22.29 & 2.57 & 0.75 \\
\hline 2 & 16.47 & 0.85 & 24.77 & 2.76 & 0.81 \\
\hline 3 & 10.81 & 0.56 & 16.25 & 2.29 & 0.67 \\
\hline 4 & 14.43 & 0.74 & 21.70 & 2.07 & 0.60 \\
\hline 5 & 9.22 & 0.47 & 13.86 & 2.03 & 0.59 \\
\hline 6 & 26.91 & 1.38 & 40.46 & 3.59 & 1.05 \\
\hline 7 & 74.44 & 3.83 & 111.93 & 4.18 & 1.22 \\
\hline 8 & 25.01 & 1.29 & 37.61 & 3.64 & 1.06 \\
\hline 9 & 19.79 & 1.02 & 29.75 & 2.54 & 0.74 \\
\hline 10 & 16.87 & 0.87 & 25.37 & 2.24 & 0.65 \\
\hline 11 & 22.13 & 1.14 & 33.27 & 2.48 & 0.72 \\
\hline 12 & 68.81 & 3.54 & 103.46 & 4.63 & 1.35 \\
\hline 13 & 77.83 & 4.01 & 117.03 & 5.42 & 1.58 \\
\hline 14 & 38.03 & 1.96 & 57.18 & 4.22 & 1.23 \\
\hline 15 & 25.97 & 1.34 & 39.05 & 2.73 & 0.80 \\
\hline 16 & 27.73 & 1.43 & 41.69 & 2.61 & 0.76 \\
\hline 17 & 66.21 & 3.41 & 99.55 & 3.85 & 1.12 \\
\hline 18 & 97.44 & 5.01 & 146.50 & 5.63 & 1.64 \\
\hline 19 & 91.71 & 4.72 & 137.89 & 4.91 & 1.43 \\
\hline 20 & 31.03 & 1.60 & 46.66 & 2.93 & 0.85 \\
\hline 21 & 40.32 & 2.08 & 60.63 & 3.40 & 0.99 \\
\hline 22 & 145.17 & 7.47 & 218.27 & 5.88 & 1.72 \\
\hline 23 & 202.96 & 10.44 & 305.17 & 6.81 & 1.99 \\
\hline 24 & 64.85 & 3.34 & 97.50 & 3.25 & 0.95 \\
\hline 25 & 22.44 & 1.15 & 33.74 & 2.38 & 0.69 \\
\hline 26 & 38.45 & 1.98 & 57.81 & 2.73 & 0.80 \\
\hline 27 & 174.01 & 8.95 & 261.64 & 5.01 & 1.46 \\
\hline 28 & 110.14 & 5.67 & 165.60 & 4.92 & 1.44 \\
\hline 29 & 76.56 & 3.94 & 115.11 & 3.93 & 1.15 \\
\hline 30 & 71.07 & 3.66 & 106.86 & 2.56 & 0.75 \\
\hline 31 & 36.16 & 1.86 & 54.37 & 2.58 & 0.75 \\
\hline 32 & 170.50 & 8.77 & 256.36 & 4.91 & 1.44 \\
\hline 33 & 64.95 & 3.34 & 97.65 & 4.72 & 1.38 \\
\hline 34 & 59.27 & 3.05 & 89.12 & 6.75 & 1.97 \\
\hline 35 & 38.24 & 1.97 & 57.50 & 3.14 & 0.92 \\
\hline 36 & 118.58 & 6.10 & 178.29 & 3.52 & 1.03 \\
\hline 37 & 113.87 & 5.86 & 171.21 & 5.16 & 1.51 \\
\hline 38 & 86.84 & 4.47 & 130.57 & 9.61 & 2.81 \\
\hline 39 & 73.34 & 3.77 & 110.27 & 8.03 & 2.35 \\
\hline 40 & 32.85 & 1.69 & 49.39 & 2.79 & 0.82 \\
\hline 41 & 15.31 & 0.79 & 23.02 & 2.60 & 0.76 \\
\hline 42 & 60.13 & 3.09 & 90.40 & 3.45 & 1.01 \\
\hline 43 & 290.90 & 14.97 & 437.38 & 10.97 & 3.20 \\
\hline 44 & 178.02 & 9.16 & 267.67 & 67.35 & 19.68 \\
\hline 45 & 79.69 & 4.10 & 119.82 & 6.51 & 1.90 \\
\hline 46 & 40.40 & 2.08 & 60.74 & 2.85 & 0.83 \\
\hline 47 & 40.85 & 2.10 & 61.42 & 3.47 & 1.01 \\
\hline 48 & 90.88 & 4.68 & 136.64 & 10.99 & 3.21 \\
\hline 49 & 228.49 & 11.76 & 343.54 & 7.74 & 2.26 \\
\hline 50 & 45.07 & 2.32 & 67.77 & 4.79 & 1.40 \\
\hline 51 & 22.76 & 1.17 & 34.23 & 2.54 & 0.74 \\
\hline
\end{tabular}


TABLE 2: Continued.

\begin{tabular}{|c|c|c|c|c|c|}
\hline Box & $\begin{array}{l}S_{\mathrm{CO}(2-1)} \Delta V \\
\left(\mathrm{Jy} \mathrm{km} \mathrm{s}^{-1}\right) \\
\end{array}$ & $\begin{array}{c}M\left(\mathrm{H}_{2}\right) \\
M_{\odot} \times 10^{6}\end{array}$ & $\begin{array}{c}\sum \mathrm{H}_{2} \\
\left(M_{\odot} \mathrm{pc}^{-2}\right)\end{array}$ & $\begin{array}{c}\text { SFR } \\
\left(M_{\odot} \mathrm{yr}^{-1}\right) \times 10^{-5} \\
\end{array}$ & $\begin{array}{c}\sum \mathrm{SFR} \\
\left(M_{\odot} \mathrm{yr}^{-1} \mathrm{kpc}^{-2}\right) \times 10^{-3}\end{array}$ \\
\hline 52 & 74.14 & 3.82 & 111.47 & 5.67 & 1.66 \\
\hline 53 & 147.76 & 7.60 & 222.17 & 6.43 & 1.88 \\
\hline 54 & 27.92 & 1.44 & 41.98 & 3.46 & 1.01 \\
\hline 55 & 36.12 & 1.86 & 54.31 & 4.08 & 1.19 \\
\hline 56 & 182.48 & 9.39 & 274.37 & 5.63 & 1.64 \\
\hline 57 & 49.71 & 2.56 & 74.74 & 3.54 & 1.04 \\
\hline 58 & 92.37 & 4.75 & 138.89 & 4.67 & 1.37 \\
\hline 59 & 103.44 & 5.32 & 155.53 & 5.84 & 1.71 \\
\hline 60 & 47.43 & 2.44 & 71.32 & 4.17 & 1.22 \\
\hline 61 & 19.06 & 0.98 & 28.66 & 2.84 & 0.83 \\
\hline 62 & 50.73 & 2.61 & 76.28 & 4.29 & 1.25 \\
\hline 63 & 115.24 & 5.93 & 173.27 & 5.95 & 1.74 \\
\hline 64 & 65.64 & 3.38 & 98.69 & 5.83 & 1.70 \\
\hline 65 & 36.63 & 1.88 & 55.08 & 3.57 & 1.04 \\
\hline 66 & 14.23 & 0.73 & 21.39 & 2.54 & 0.74 \\
\hline 67 & 53.09 & 2.73 & 79.82 & 4.12 & 1.20 \\
\hline 68 & 85.09 & 4.38 & 127.93 & 5.41 & 1.58 \\
\hline 69 & 95.66 & 4.92 & 143.84 & 6.73 & 1.97 \\
\hline 70 & 28.83 & 1.48 & 43.35 & 3.74 & 1.09 \\
\hline 71 & 30.92 & 1.59 & 46.49 & 2.74 & 0.80 \\
\hline 72 & 37.06 & 1.91 & 55.73 & 3.73 & 1.09 \\
\hline 73 & 58.65 & 3.02 & 88.18 & 4.30 & 1.26 \\
\hline 74 & 24.50 & 1.26 & 36.83 & 3.17 & 0.93 \\
\hline 75 & 16.90 & 0.87 & 25.40 & 2.04 & 0.60 \\
\hline 76 & 20.26 & 1.04 & 30.45 & 2.32 & 0.68 \\
\hline
\end{tabular}

Note. Column 1: region name. Column 2: intensity of ${ }^{12} \mathrm{CO}(J=2-1)$ emission. Column 3: molecular gas mass. Column 4: surface density of molecular hydrogen mass. Column 5: star formation rate. Column 6: Star formation rate surface density.

a time period of $10 \mathrm{Myr}$. The drift scale of $100 \mathrm{pc}$ is close to our scale of $185 \mathrm{pc}$. This result indicates that the traditional K-S law in this elliptical galaxy holds at greater than $200 \mathrm{pc}$; thus, when the spatial resolution is below this value, the K-S law for this galaxy may be not valid. As proposed by Calzetti et al. [49] at such scales below 500 pc, the sampling effects are more clear than they are for larger scales. More thorough studies are needed to figure out the effect of sampling effects versus scales, especially for the high spatial resolution (small region size). In addition the type of fitting method has shown to have an effect on the results of star formation law as proved by Rahmani et al. [50], so it will be more worthy to try different fitting methods at high spatial resolutions. We also compared the CO masses with the SFR estimates in order to measure a star formation efficiency (depletion time). Using a conversion factor $X_{\mathrm{CO}}=2 \times 10^{20} \mathrm{~cm}^{-2}\left(\mathrm{~K} \mathrm{~km} \mathrm{~s}^{-1}\right)^{-1}$ led to long depletion time $(68 \mathrm{Gyr})$, which indicates that the star formation is inefficient in this galaxy. It is clear that the depletion time of the molecular gas in this galaxy is longer than the Hubble time. This means a very low efficiency of forming stars. The efficiency may also depend on the pressure and the surface density of star (e.g., [51]), which is, on average, low in this galaxy. The solid red triangle in Figure 3 represents the nuclear region of Centaurus A. It is clear that the molecular gas at this point is obviously offset from the KS law, suggesting that this points is mainly affected by AGN activities instead of star formation [17]. This means that this point is powered by kinetic energy injection from the AGN jet/wind and leading to molecular gas reservoir not forming star efficiently [52].

Figure 4 shows our elliptical galaxy, Centaurus A, and the normal spiral and starburst galaxies of Kennicutt [13] on the same plot for comparison. We then went on to investigate the position of Centaurus A on the Kennicutt-Schmidt (KS) relation. We found that this galaxy has comparable star formation surface densities to normal spiral galaxy centres, but it lies systematically offset from the KS relation, having lower star formation surface density. This is in agreement with Davis et al. [53] who compared the Kennicutt-Schmidt (KS) relation [13] of nearby spiral galaxies with that of the CO-detected ETGs. They found that the ETGs had lower average SFR surface densities at a given molecular gas surface density compared to spirals. This is in agreement with recent simulations by Martig et al. [54] who predicted a decrease in the SFEs of ETGs

As we know, the velocity dispersion of the gas is also an important parameter for the star formation law [55]. We present here a measurement of the velocity dispersion 


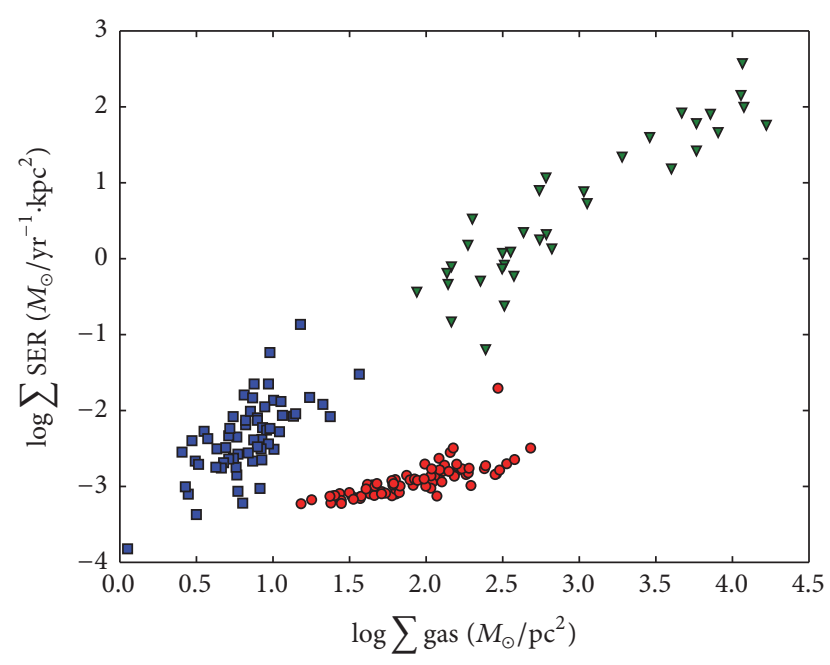

FIGURE 4: Relation between the surface density of molecular gas $\sum \mathrm{H}_{2}$ and $\sum \mathrm{SFR}$ for normal galaxies (blue squares), starburst galaxies (green triangle), and the elliptical galaxy Centaurus $\mathrm{A}$ (red circles). The gas surface density is derived from $\mathrm{CO}(2-1)$ emission. The normal and starburst galaxy samples are obtained from Kennicutt [13].

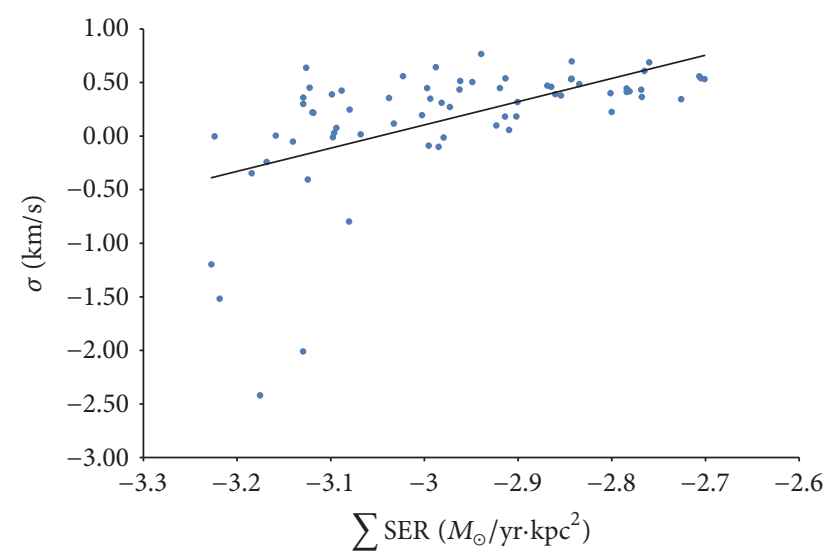

Figure 5: The velocity dispersion for each pixel $\sigma$ of Centaurus A as a function of the SFR surface averaged (in units of $M_{\odot} / \mathrm{yr} \cdot \mathrm{kpc}^{2}$ ).

for molecular components of the ISM for the outer disk of Centaurus A. In Figure 5 we investigate the correlation of the velocity dispersion $\sigma$ of molecular gas with the star formation rate surface density $\sum$ SFR of the galaxy. The former one is estimated from the second moments map (Figure 2) of the galaxy using the data from ALMA. The velocity dispersion in outer disk ranges from 0.02 to $31.50 \mathrm{~km} / \mathrm{s}$ with an average value of $11.78 \mathrm{~km} / \mathrm{s}$. This average value of velocity dispersion is consistent with previous measurements for the Milky Way [56], M33 [57], and other nearby galaxies [58-60]. We find a significant correlation coefficient $r=0.59$ with probability $p=99.99 \%$. Generally the results indicate low values for the velocity dispersion. According to Krumholz \& Dekel [61] the low velocity dispersion values are usually associated with low SFR values. The determined average value which is close to 10 indicates low star formation for this elliptical galaxy. This indicates that there is no gravitational collapse, accordingly there is no star formation. We can see from Figure 5 that the velocity dispersion of the molecular ISM for the outer disk follows a power relation $\sigma \propto\left(\sum \mathrm{SFR}\right)^{\beta}$ where $\beta$ is the slope from the ordinary least square fitting. The value of $\beta$ is found to $b e \approx 1 / n=2.16 \pm 0.4$ and $n$ is the power low index of the star formation law. This power law correlation may be a natural consequence of the gas and star formation surface density scaling laws [62].

\section{Summary and Conclusions}

We used a high spatial resolution mosaic of ALMA observations of the ${ }^{12} \mathrm{CO}(J=2-1)$ line emission as a molecular gas indicator in the central region of the elliptical galaxy Centaurus A (NGC 5128) at a distance of $3.8 \mathrm{Mpc}\left(1^{\prime \prime}=\right.$ $18.5 \mathrm{pc}$ ). We measured $\sum$ SFR with $24 \mu \mathrm{m}$ data from the (MIPS) Spitzer Space Telescope. We investigated the SF law (SFL) for this elliptical galaxy at very high spatial resolution $(185 \mathrm{pc})$, and the result showed a breakdown SFL at this spatial resolution. The best fit parameter for the star formation power law index is $0.49 \pm 0.05$. The second moment map from ALMA data for this galaxy was used to find the velocity dispersion of the molecular component for the interstellar medium. The results for the average velocity dispersion are close to $10 \mathrm{kms}^{-1}$ which is associated with low star formation, due to no gravitational collapse, which leads to no star formation. This result is supported by the long depletion time (68 Gyr) (low star formation efficiency). In addition, we find a significant correlation between velocity dispersion for each pixel and star formation surface density which follows a power law relation $\sigma \propto\left(\sum \mathrm{SFR}\right)^{\beta}$, where the value of $\beta$ is approximately $\approx 1 / n$ and $n$ is set by the Kennicutt-Schmidt law $\sum$ SFR $\propto\left(\sum \text { gas }\right)^{n}$. This power law correlation may be a natural result of the gas and star formation surface density scaling laws.

We can see that the star formation efficiency (SFE) is very low in this galaxy despite the fact that the amount of molecular gas reservoir available is huge when compared to standard star formation efficiencies. This suggests that some processes may prevent the star formation to proceed in the cold gas. These processes can be due to high kinetic energy injection from the AGN that suppress the star formation processes [44].

\section{Conflicts of Interest}

The authors declare that they have no conflicts of interest.

\section{Acknowledgments}

This paper makes use of the following ALMA data: ADS/JAO.ALMA\#2011.0.00008.SV. ALMA is a partnership of ESO (representing its member states), NSF (USA), and NINS (Japan), together with NRC (Canada) and NSC and ASIAA (Taiwan), and KASI (Republic of Korea), in cooperation with the Republic of Chile. The Joint ALMA Observatory is operated by ESO, AUI/NRAO, and NAOJ. The coauthor Zamri 
Z. Abidin would also like to acknowledge the University of Malaya's HIR Grant UM.S/625/3/HIR/28 for their funding.

\section{References}

[1] T. Wiklind, F. Combes, C. Henkel, and F. Wyrowski, "Molecular gas in the elliptical galaxy NGC 759: Interferometric CO observations," Astronomy and Astrophysics, vol. 323, no. 3, pp. 727-738, 1997.

[2] S. K. Yi, S.-J. Yoon, S. Kaviraj et al., "Galaxy Evolution Explorer ultraviolet color-magnitude relations and evidence of recent star formation in early-type galaxies," The Astrophysical Journal Letters, vol. 619, p. L111, 2005.

[3] R. Morganti, P. T. De Zeeuw, T. A. Oosterloo et al., "Neutral hydrogen in nearby elliptical and lenticular galaxies: the continuing formation of early-type galaxies," Monthly Notices of the Royal Astronomical Society, vol. 371, no. 1, pp. 157-169, 2006.

[4] S. Kaviraj, K. Schawinski, J. E. G. Devriendt et al., "UVoptical colors as probes of early-type galaxy evolution," The Astrophysical Journal: Supplement Series, vol. 173, no. 2, p. 619, 2007.

[5] M. Sarzi, R. Bacon, M. Cappellari et al., "Recent star formation in nearby early-type galaxies," in Pathways Through an Eclectic Universe, J. H. Knapen, T. J. Mahoney, and A. Vazdekis, Eds., vol. 390 of Astronomical Society of the Pacific Conference Series, p. 218, 2008.

[6] S. Onodera, N. Kuno, T. Tosaki et al., "Breakdown of kennicutt-Schmidt law at giant molecular cloud scales in M33," The Astrophysical Journal Letters, vol. 722, p. L127, 2010.

[7] L. H. Wei, S. N. Vogel, S. J. Kannappan, A. J. Baker, D. V. Stark, and S. Laine, "The relationship between molecular gas and star formation in low-mass E/S0 galaxies," Astrophysical Journal Letters, vol. 725, no. 1, pp. L62-L67, 2010.

[8] L. M. Young, M. Bureau, T. A. Davis et al., "The ATLAS ${ }^{3 D}$ project - IV. The molecular gas content of early-type galaxies," Monthly Notices of the Royal Astronomical Society, vol. 414, no. 2, pp. 940-967, 2011.

[9] A. Crocker, M. Krips, M. Bureau et al., "The ATLAS ${ }^{3 D}$ project XI. Dense molecular gas properties of CO-luminous early-type galaxies," Monthly Notices of the Royal Astronomical Society, vol. 421, no. 2, pp. 1298-1314, 2012.

[10] T. A. Davis, K. Rowlands, J. R. Allison et al., "Molecular and atomic gas in dust lane early-type galaxies - I. Low star formation efficiencies in minor merger remnants," Monthly Notices of the Royal Astronomical Society, vol. 449, no. 4, pp. 3503-3516, 2015.

[11] M. Schmidt, “The Rate of Star Formation," Astrophysical Journal, vol. 129, p. 243, 1959.

[12] R. C. Kennicutt Jr., "Star formation in galaxies along the hubble sequence," Annual Review of Astronomy and Astrophysics, vol. 36, pp. 189-231, 1998.

[13] R. C. Kennicutt Jr., “The global schmidt law in star-forming galaxies," The Astrophysical Journal, vol. 498, p. 541, 1998.

[14] F. Bigiel, A. Leroy, F. Walter et al., "The star formation law in nearby galaxies on SUB-KPC scales," Astronomical Journal, vol. 136, no. 6, pp. 2846-2871, 2008.

[15] F. Bigiel, A. K. Leroy, F. Walter et al., "A Constant Molecular Gas Depletion Time in Nearby Disk Galaxies," The Astrophysical Journal Letters, vol. 730, p. L13, 2011.

[16] A. K. Leroy et al., "Molecular Gas and Star Formation in Nearby Disk Galaxies," The Astronomical Journal, vol. 146, p. 19, 2013.
[17] M. Tsai, C.-Y. Hwang, S. Matsushita, A. J. Baker, and D. Espada, "Interferometric $\mathrm{CO}(32)$ observations toward the central region of NGC1068," Astrophysical Journal, vol. 746, no. 2, article no. 129, 2012.

[18] R. C. Kennicutt and N. J. Evans, "Star Formation in the Milky Way and Nearby Galaxies," Annual Review of Astronomy and Astrophysics, vol. 50, pp. 531-608, 2012.

[19] C. F. McKee and E. C. Ostriker, "Theory of star formation," Annual Review of Astronomy and Astrophysics, vol. 45, pp. 565687, 2007.

[20] F. P. Israel, "Centaurus A - NGC 5128," The Astronomy and Astrophysics Review, vol. 8, pp. 237-278, 1998.

[21] N. Neumayer, M. Cappellari, J. Reunanen et al., "The central parsecs of centaurus A: High-excitation gas, a molecular disk, and the mass of the black hole," Astrophysical Journal, vol. 671, no. 2, pp. 1329-1344, 2007.

[22] I. D. Karachentsev, "The local group and other neighboring galaxy groups," Astronomical Journal, vol. 129, no. 1, pp. 178-188, 2005.

[23] G. L. H. Harris, M. Rejkuba, and W. E. Harris, "The distance to NGC 5128 (Centaurus A)," Publications of the Astronomical Society of Australia, vol. 27, no. 4, pp. 457-462, 2010.

[24] M. Rejkuba, W. E. Harris, L. Greggio, and G. L. H. Harris, "How old are the stars in the halo of NGC 5128 (Centaurus A)?" Astronomy \& Astrophysics, vol. 526, p. A123, 2011.

[25] J. Ott, M. McCoy, and D. Meier, "IAU Symposium," in IAU Symposium, T. Wong and J. Ott, Eds., vol. 292, pp. 251-251, 2013.

[26] J. E. Barnes, "Formation of gas discs in merging galaxies," Monthly Notices of the Royal Astronomical Society, vol. 333, no. 3, pp. 481-494, 2002.

[27] R. Auld, M. W. L. Smith, G. Bendo et al., "Herschel observations of Cen A: stellar heating of two extragalactic dust clouds," Monthly Notices of the Royal Astronomical Society, vol. 420, no. 3, pp. 1882-1896, 2012.

[28] T. G. Phillips, B. N. Ellison, J. B. Keene et al., "CO emission from Centaurus A," Astrophysical Journal, Part 2, vol. 322, p. L73, 1987.

[29] A. Eckart, M. Cameron, H. Rothermel et al., "Observations of $\mathrm{CO}$ isotopic emission and the far-infrared continuum of centaurus A," Astrophysical Journal, vol. 363, no. 2, pp. 451-463, 1990.

[30] A. Eckart, M. Cameron, R. Genzel et al., "Molecular absorption lines toward the nucleus of Centaurus A," Astrophysical Journal, vol. 365, no. 2, pp. 522-531, 1990.

[31] F. P. Israel, E. F. van Dishoeck, F. Baas, J. Koornneef, J. H. Black, and T. de Graauw, "H2 emission and $\mathrm{CO}$ absorption in Centaurus A - evidence for a circumnuclear molecular disk," Astronomy \& Astrophysics, vol. 227, p. 342, 1990.

[32] F. P. Israel, E. F. van Dishoeck, F. Baas, T. de Graauw, and T. G. Phillips, "CO J = 1-0, 2-1 and 3-2 absorption and emission toward the nucleus of centaurus A(asterisk) - probing the circumnuclear disk," Astronomy \& Astrophysics, vol. 245, p. L13, 1991.

[33] A. C. Quillen, P. T. de Zeeuw, E. S. Phinney, and T. G. Phillips, "The kinematics of the molecular gas in Centaurus A," Astrophysical Journal, Part 1, vol. 391, p. 121, 1992.

[34] G. Rydbeck, T. Wiklind, M. Cameron et al., "High resolution (C-12) $\mathrm{O}(2-1)$ observations of the molecular gas in centaurus A," Astronomy \& Astrophysics, vol. 270, p. L13, 1993.

[35] V. Charmandaris, F. Combes, and J. M. van der Hulst, "First detection of molecular gas in the shells of CenA," Astronomy \& Astrophysics, vol. 356, p. L1, 2000. 
[36] J. Ott, D. S. Meier, M. McCoy et al., "Discovery of nuclear water maser emission in Centaurus A," The Astrophysical Journal Letters, vol. 771, p. L41, 2013.

[37] K. Ebneter and B. Balick, "Centaurus A," Publications of the Astronomical Society of the Pacific, vol. 95, p. 675, 1983.

[38] J. Bland, k. Taylor, and P. D. Atherton, "The structure and kinematics of the ionized gas within NGC 5128 (Cen A) - I. TAURUS observations," Monthly Notices of the Royal Astronomical Society, vol. 228, no. 3, pp. 595-621, 1987.

[39] J. S. Young, Star Forming Regions, Springer Netherlands, Dordrecht, 1987.

[40] A. K. Leroy, F. Walter, F. Bigiel et al., "Heracles: the hera co line extragalactic survey," The Astronomical Journal, vol. 137, p. 4670, 2009.

[41] R. Morganti, T. Oosterloo, J. B. Raymond Oonk, F. Santoro, and C. Tadhunter, "The missing link: tracing molecular gas in the outer filament of Centaurus A," Astronomy \& Astrophysics, vol. 592, p. L9, 2016.

[42] D. Espada, S. Matsushita, A. Pecket et al., "Disentangling the circumnuclear environs of centaurus A. I. High-resolution molecular gas imaging," The Astrophysical Journal, vol. 695, p. 116, 2009.

[43] J. H. Azeez, C.-Y. Hwang, Z. Z. Abidin, and Z. A. Ibrahim, "Kennicutt-Schmidt law in the central region of NGC 4321 as seen by ALMA," Scientific Reports, vol. 6, p. 26896, 2016.

[44] Q. Salomé, P. Salomé, F. Combes, and S. Hamer, "Atomic-tomolecular gas phase transition triggered by the radio jet in Centaurus A," Astronomy \& Astrophysics, vol. 595, p. A65, 2016.

[45] G. Helou, “The IRAS colors of normal galaxies," Astrophysical Journal, vol. 311, pp. L33-L36, 1986.

[46] G. J. Bendo, F. Galliano, and S. C. Madden, "MIPS 24-160 $\mu \mathrm{m}$ photometry for the Herschel-SPIRE local galaxies guaranteed time programs," Monthly Notices of the Royal Astronomical Society, vol. 423, no. 1, pp. 197-212, 2012.

[47] H. Wu, C. Cao, C.-N. A. Hao et al., "PAH and MID-infrared luminosities as measures of star formation rate in spitzer first look survey galaxies," Astrophysical Journal, vol. 632, no. 2, pp. L79-L82, 2005.

[48] D. Leisawitz, F. N. Bash, and P. Thaddeus, "A CO survey of regions around 34 open clusters," Astrophysical Journal: Supplement Series, vol. 70, pp. 731-812, 1989.

[49] D. Calzetti, G. Liu, and J. Koda, "Star formation laws: The effects of gas cloud sampling," Astrophysical Journal, vol. 752, no. 2, article 98, 2012.

[50] S. Rahmani, S. Lianou, and P. Barmby, "Star formation laws in the Andromeda galaxy: gas, stars, metals and the surface density of star formation," Monthly Notices of the Royal Astronomical Society, vol. 456, no. 4, pp. 4128-4144, 2016.

[51] L. Blitz and E. Rosolowsky, "The role of pressure in GMC formation II: The H2-pressure relation," Astrophysical Journal, vol. 650, no. 2 I, pp. 933-944, 2006.

[52] Q. Salomé, P. Salomé, F. Combes, S. Hamer, and I. Heywood, "Star formation efficiency along the radio jet in Centaurus A," Astronomy \& Astrophysics, vol. 586, p. A45, 2016.

[53] T. A. Davis, L. M. Young, A. F. Crocker et al., "The ATLAS3D Project - XXVIII. Dynamically driven star formation suppression in early-type galaxies," Monthly Notices of the Royal Astronomical Society, vol. 444, no. 4, pp. 3427-3445, 2014.

[54] M. Martig et al., "The ATLAS3D project - XXII. Low-efficiency star formation in early-type galaxies: hydrodynamic models and observations," Monthly Notices of the Royal Astronomical Society, vol. 432, 2013.
[55] R. C. Kennicutt Jr., "The star formation law in galactic disks," Astrophysical Journal, Part 1, vol. 344, pp. 685-703, 1989.

[56] A. A. Stark and J. Brand, "Kinematics of molecular clouds. II - New data on nearby giant molecular clouds," Astrophysical Journal, Part 1, vol. 339, pp. 763-771, 1989.

[57] C. D. Wilson and N. Z. Scoville, "The properties of individual giant molecular clouds in M33," Astrophysical Journal, Part 1, vol. 363, pp. 435-450, 1990.

[58] F. Combes and J.-F. Becquaert, "Perspectives for detecting cold H2 in outer galactic disks," Astronomy \& Astrophysics, vol. 327, p. 453, 1997.

[59] W. Walsh, R. Beck, G. Thuma, A. Weiss, R. Wielebinski, and M. Dumke, "Molecular gas in NGC 6946," Astronomy and Astrophysics, vol. 388, no. 1, pp. 7-28, 2002.

[60] C. D. Wilson, B. E. Warren, J. Irwin et al., "The JCMT Nearby Galaxies Legacy Survey - IV. Velocity dispersions in the molecular interstellar medium in spiral galaxies," Monthly Notices of the Royal Astronomical Society, vol. 410, no. 3, pp. 1409-1422, 2011.

[61] M. R. Krumholz and A. Dekel, "Survival of star-forming giant clumps in high-redshift galaxies," Monthly Notices of the Royal Astronomical Society, vol. 406, no. 1, pp. 112-120, 2010.

[62] A. M. Swinbank, I. Smail, D. Sobral, T. Theuns, P. N. Best, and J. E. Geach, "The properties of the star-forming interstellar medium at $z=0.8-2.2$ from HiZELS: star formation and clump scaling laws in gas-rich, turbulent disks," Astrophysical Journal, vol. 760, no. 2, article 130, 2012. 

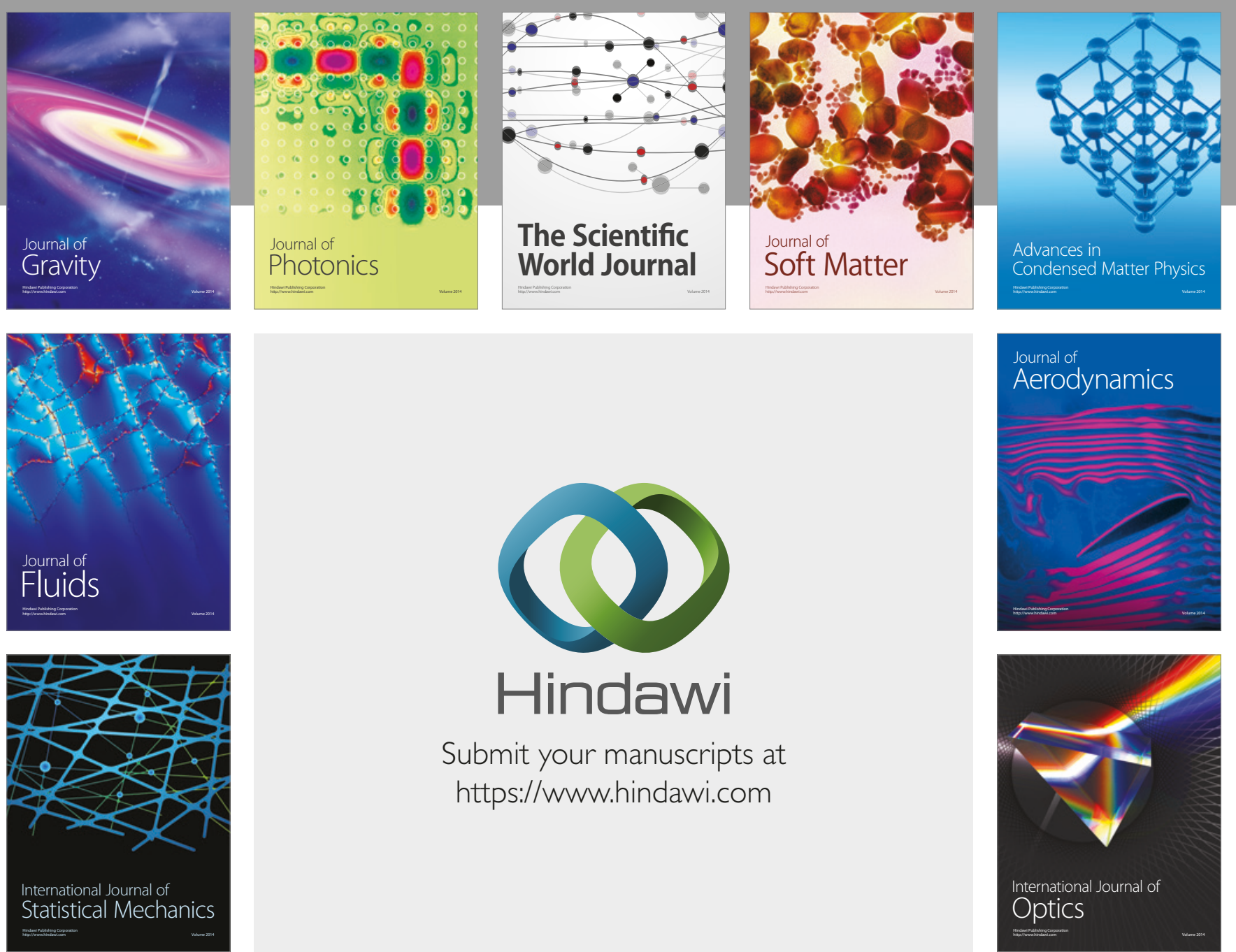

Submit your manuscripts at

https://www.hindawi.com
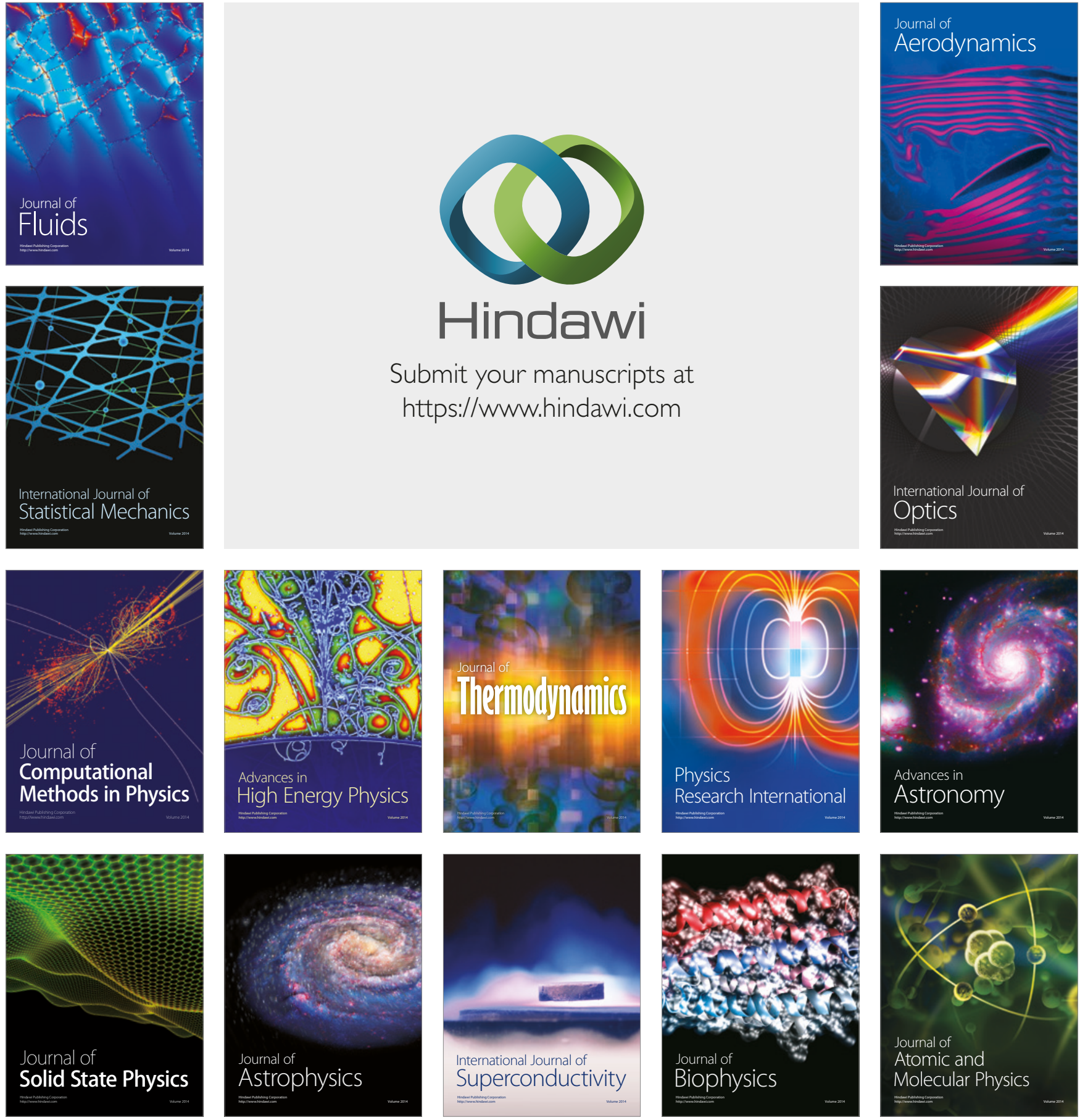